JOURNAL OF

FUNCTION SPACES AND APPLICATIONS

Volume 8, Number 3 (2010), 245-256 (c) 2010, Scientific Horizon http://www.jfsa.net

\title{
The Boundedness of Commutators of Singular Integral Operators with Besov Functions ${ }^{1}$
}

\author{
Xionglue Gao and Bolin Ma \\ (Communicated by Vladimir D. Stepanov)
}

2000 Mathematics Subject Classification. 42B20 , 42B25.

Keywords and phrases. Singular Integral Operator; Commutator; Besov Function.

\footnotetext{
Abstract. In this paper, we prove the boundedness of commutator generated by singular integral operator and Besov function from some $L^{d}$ to Triebel-Lizorkin spaces.
}

\section{Introduction}

Let $K$ be a Calderón-Zygmund singular integral kernel being of the form

$$
K(x)=\frac{\Omega(x)}{|x|^{n}},
$$

where $\Omega \in C^{\infty}\left(\Sigma^{n-1}\right)$ is homogeneous of degree 0 and

$$
\int_{\Sigma^{n-1}} \Omega(x) d x=0 .
$$

\footnotetext{
${ }^{1}$ The work is partially supported by NSF of China (10771054) .
} 
The Calderón-Zygmund singular integral operator $T$ is defined by

$$
T f(x)=\text { p.v. } \int_{\mathbb{R}^{n}} K(x-y) f(y) d y .
$$

The fractional integral operator $I^{\alpha}$ of order $\alpha, 0<\alpha<n$, is the convolution operator with the kernel $\omega_{\alpha}|x|^{\alpha-n}$. In [3], a well known result of Coifman, Rochberg and Weiss states that the commutator of singular integral operator

$$
[b, T] f(x)=b(x) T f(x)-T(b f)(x)
$$

is bounded on some $L^{p}, 1<p<\infty$, if and only if $b \in$ BMO . In 1982, Chanillo [1] introduced the commutator of fractional integral operator $\left[b, I^{\alpha}\right]$ :

$$
\left[b, I^{\alpha}\right] f(x)=b(x) I^{\alpha} f(x)-I^{\alpha}(b f)(x),
$$

and proved a similar result when $\mathrm{T}$ is replaced by the fractional integral operator. In [5], Janson investigated the commutator of singular integral operator and fractional integral operator with Lipschitz functions. In 1995, Paluszynski [6] proved that $[b, T]$ is bounded from $L^{p}$ to $F_{p}^{\beta, \infty}$, the TriebelLizorkin space, if and only if $b \in \dot{\Lambda}_{\beta}$, where $\dot{\lambda}_{\beta}$ is the homogeneous Lipschitz space which is the set of functions satisfing

$$
\|f\|_{\dot{\lambda}_{\beta}}=\sup _{\substack{x, h \in R^{n} \\ h \neq 0}} \frac{\left|\Delta_{h}^{[\beta]+1} f(x)\right|}{|h|^{\beta}}<\infty
$$

where $\Delta_{h}^{k}$ denotes the $k$-th difference operator (see [6]).

A generalization of Lipschitz sapce is Besov space $\dot{\lambda}_{\beta}^{p, q}\left(R^{n}\right)$ defined by the set of all functions satisfying

$$
\|b\|_{\lambda_{\beta}^{p, q}}=\left(\int_{R^{n}} \frac{\|b(x+t)-b(x)\|_{p}^{q}}{|t|^{n+\beta q}} d t\right)^{1 / q}<\infty .
$$

For the properties of Besov functions we refer [9]. In 2006, Y. Chen and B. Ma in [2] introduced the commutator of singular integral operators and Besov functions and proved that $[b, T]^{k}$ is bounded from some $L^{d}$ to certain $L^{r}$.

In this paper, we investigate the boundedness of commutators generated by singular integral operators and Besov functions from Lebesgue integrable spaces to Triebel-Lizorkin spaces, as well as commutators of fractional 
integral operators. We simply denote $T_{b}$ by $[b, T]$ and $I_{b}^{\alpha}$ by $\left[b, I^{\alpha}\right]$. Our results are stated below.

Theorem 1.1. Let $1<q<p<\infty, 0<\beta-n / p<1$ and $b \in \dot{\Lambda}_{\beta}^{p, q}$. If $d>q /(q-1)$, then the commutator $T_{b}$ is a bounded operator from $L^{d}\left(\mathbb{R}^{n}\right)$ to $\dot{F}_{d}^{(\beta-n / p), \infty}\left(\mathbb{R}^{n}\right)$.

Theorem 1.2. Let $1<p<q<\infty, 0<\beta-n / p<1$ and $b \in \dot{\Lambda}_{\beta}^{p, q}$. If $d>p /(p-1)$, then the commutator $T_{b}$ is a bounded operator from $L^{d}\left(\mathbb{R}^{n}\right)$ to $\dot{F}_{d}^{(\beta-n / p), \infty}\left(\mathbb{R}^{n}\right)$.

Theorem 1.3. Let $1<q<p<\infty, 0<\beta-n / p<1, b \in \dot{\Lambda}_{\beta}^{p, q}$ and $0<\alpha<n$. If $d>q /(q-1), 1 / d-1 / r=\alpha / n$, then the commutator $I_{b}^{\alpha} f$ is a bounded operator from $L^{d}\left(\mathbb{R}^{n}\right)$ to $\dot{F}_{r}^{(\beta-n / p), \infty}\left(\mathbb{R}^{n}\right)$.

Theorem 1.4. Let $1<p<q<\infty, 0<\beta-n / p<1, b \in \dot{\wedge}_{\beta}^{p, q}$ and $0<\alpha<n$. If $d>p /(p-1), 1 / d-1 / r=\alpha / n$, then the commutator $I_{b}^{\alpha}$ is a bounded operator from $L^{d}\left(\mathbb{R}^{n}\right)$ to $\dot{F}_{r}^{(\beta-n / p), \infty}\left(\mathbb{R}^{n}\right)$.

From the results stated above it can be seen that if $p, q \rightarrow \infty$ then all the theorems are the ones obtained by Paluszynski in [6]. The proofs of our theorems are based on an estimate similar to the mean oscillation of a Besov function over cubes. We shall provide it as a lemma in the next section and then prove our theorems.

\section{Some Lemmas and Proofs of Results}

In this section, we get an estimate of the oscillation of Besov function $b$ on cubes. Using the property of Besov function, we discuss the boundedness of the commutator. In the following, let $Q$ be a cube and denote by $f_{Q}$ the mean of $f$ over $Q$, i.e.,

$$
f_{Q}=\frac{1}{|Q|} \int_{Q} f(y) \mathrm{d} y
$$

Lemma 2.1. ([6]) For $0<\beta<1,1<p<\infty$, we have

$$
\|f\|_{\dot{F}_{p}^{\beta, \infty}} \approx\left\|\sup _{Q} \frac{1}{|Q|^{1+\beta / n}} \int_{Q}\left|f-f_{Q}\right|\right\|_{p} .
$$


Lemma 2.2. (a) For $0<\beta<1,1<q \leq p<\infty$, we have

$$
\begin{aligned}
\sup _{Q} & \frac{1}{|Q|^{1+\beta / n-1 / p}} \int_{Q}\left|b(y)-b_{Q}\right| \mathrm{d} y \\
& \leq \sup _{Q} \frac{1}{|Q|^{\beta / n+1 / q-1 / p}}\left(\int_{Q}\left|b(y)-b_{Q}\right|^{q} \mathrm{~d} y\right)^{1 / q} \leq C\|b\|_{i_{\beta}^{p, q}}
\end{aligned}
$$

(b) For $0<\beta<1,1<p \leq q<\infty$, we have:

$$
\sup _{Q} \frac{1}{|Q|^{\beta / n}}\left(\int_{Q}\left|b(y)-b_{Q}\right|^{p} \mathrm{~d} y\right)^{1 / p} \leq C\|b\|_{\lambda_{\beta}^{p, q} .}
$$

Proof. Fix a cube Q, and set $\wedge=\{t=y-z: y, z \in Q\}$. So for any $t \in \wedge$, we have $|t| \leq C|Q|^{1 / n}$. Using Hölder's inequality, we first estimate $\int_{Q}\left|b(y)-b_{Q}\right|^{q} \mathrm{~d} y$ as follows

$$
\begin{aligned}
\int_{Q}\left|b(y)-b_{Q}\right|^{q} \mathrm{~d} y & =\int_{Q}\left|b(y)-\frac{1}{|Q|} \int_{Q}\right| b(z)|\mathrm{d} z|^{q} \mathrm{~d} y \\
& \leq \int_{Q}\left(\frac{1}{|Q|} \int_{Q}|b(y)-b(z)| \mathrm{d} z\right)^{q} \mathrm{~d} y \\
& \leq \int_{Q}\left(\frac{1}{|Q|} \int_{Q}|b(y)-b(z)|^{q} \mathrm{~d} z\right)\left(\frac{1}{|Q|} \int_{Q} \mathrm{~d} z\right)^{q-1} \mathrm{~d} y \\
& \leq|Q|^{-1} \int_{Q} \int_{Q}|b(y)-b(z)|^{q} \mathrm{~d} z \mathrm{~d} y \\
& \leq C|Q|^{-1} \int_{t \in \wedge} \int_{Q}|b(z+t)-b(z)|^{q} \mathrm{~d} z \mathrm{~d} t \\
& =: S_{1} .
\end{aligned}
$$

Since $p / q>1$, using Hölder's inequality on $z$, we obtain

$$
\begin{aligned}
S_{1} & \leq C|Q|^{-1} \int_{t \in \wedge}\left(\int_{Q}|b(z+t)-b(z)|^{p} \mathrm{~d} z\right)^{q / p} \mathrm{~d} t|Q|^{1-q / p} \\
& \leq C|Q|^{-1} \int_{t \in \wedge} \frac{\left(\int_{Q}|b(z+t)-b(z)|^{p} \mathrm{~d} z\right)^{q / p}}{|t|^{n+\beta q}}|t|^{n+\beta q} \mathrm{~d} t|Q|^{1-q / p} \\
& \leq C|Q|^{(-1+1-q / p)}|Q|^{1+\beta q / n}\|b\|_{\hat{\lambda}_{\beta}^{p, q}}^{q} \\
& \leq C|Q|^{(1+q \beta / n-q / p)}\|b\|_{\hat{\lambda}_{\beta}^{p, q}}^{q} .
\end{aligned}
$$

Therefore we obtain the second inequality in (2.1). 
With regard to the first inequality in (2.1), using the second one, together with Hölder inequality, we have

$$
\begin{aligned}
\int_{Q}\left|b(y)-b_{Q}\right| \mathrm{d} y & \leq\left(\int_{Q}\left|b(y)-b_{Q}\right|^{q} \mathrm{~d} y\right)^{1 / q}|Q|^{1-1 / q} \\
& \leq C|Q|^{(1-1 / q+1 / q+\beta / n-1 / p)}\|b\|_{\lambda_{\beta}^{p, q}} \\
& \leq C|Q|^{(1+\beta / n-1 / p)}\|b\|_{\lambda_{\beta}^{p, q}}
\end{aligned}
$$

Thus, the part (a) of Lemma 2.2 is proved.

Now we consider the part (b). Clearly we have

$$
\int_{Q}\left|b(y)-b_{Q}\right|^{p} \mathrm{~d} y=\int_{Q}\left|b(y)-\frac{1}{|Q|} \int_{Q} b(z) \mathrm{d} z\right|^{p} \mathrm{~d} y .
$$

Also by Hölder's inequality, we obtain

$$
\begin{aligned}
\int_{Q}\left|b(y)-b_{Q}\right|^{p} \mathrm{~d} y & \leq \int_{Q}\left(\frac{1}{|Q|} \int_{Q}|b(y)-b(z)| \mathrm{d} z\right)^{p} \mathrm{~d} y \\
& \leq \int_{Q}\left(\frac{1}{|Q|} \int_{Q}|b(y)-b(z)|^{p} \mathrm{~d} z\right)\left(\frac{1}{|Q|} \int_{Q} \mathrm{~d} z\right)^{p-1} \mathrm{~d} y \\
& \leq|Q|^{-1} \int_{Q} \int_{Q}|b(y)-b(z)|^{p} \mathrm{~d} z \mathrm{~d} y
\end{aligned}
$$

Set $y=z+t, z=z$, by a change of variable, we get

$$
\int_{Q}\left|b(y)-b_{Q}\right|^{p} \mathrm{~d} y \leq C|Q|^{-1} \int_{t \in \wedge} \int_{Q}|b(z+t)-b(z)|^{p} \mathrm{~d} z \mathrm{~d} t=: S_{2} .
$$

Since $q / p>1$, using Hölder's inequality, we have

$$
\begin{aligned}
S_{2} & \leq C|Q|^{-1}\left(\int_{t \in \wedge}\left(\int_{Q}|b(z+t)-b(z)|^{p} \mathrm{~d} z\right)^{q / p} \mathrm{~d} t\right)^{p / q}\left(\int_{t \in \wedge} \mathrm{d} t\right)^{1-p / q} \\
& \leq C|Q|^{-p / q}\left(\int_{t \in \wedge} \frac{\left(\int_{Q}|b(z+t)-b(z)|^{p} \mathrm{~d} z\right)^{q / p}}{|t|^{n+\beta q}}|t|^{n+\beta q} \mathrm{~d} t\right)^{p / q} \\
& \leq C|Q|^{(-q / p)}|Q|^{p / q+p \beta / n}\left(\int_{\mathbb{R}^{n}} \frac{\left(\int_{Q}|b(z+t)-b(z)|^{p} \mathrm{~d} z\right)^{q / p}}{|t|^{n+\beta q}} \mathrm{~d} t\right)^{p / q} \\
& \leq C|Q|^{p \beta / n}\|b\|_{\lambda_{\beta}^{p, q}}^{p} .
\end{aligned}
$$

Thus the Lemma 2.2 is proved completely. 
We now proceed with proofs of our theorems.

Proof of the Theorem 1.1: Fix a cube $Q=Q\left(x_{Q}, s\right)$, centered at $x_{Q}$,with the side length $s$, and $x \in Q$. For $f \in L^{p}$, decompose $f$ as $f_{1}=f \chi_{2 Q}, f_{2}=f-f_{1}$. Observing that $T_{b} f=T_{\left(b-b_{Q}\right)} f$, we have

$$
\begin{aligned}
\int_{Q}\left|T_{b} f(y)-\left(T_{b} f\right)_{Q}\right| \mathrm{d} y \leq & 2 \int_{Q}\left|T_{\left(b-b_{Q}\right)} f(y)-T\left(\left(b-b_{Q}\right) f_{2}\right)\left(x_{Q}\right)\right| \mathrm{d} y \\
\leq & 2 \int_{Q}\left|\left(b(y)-b_{Q}\right) T f(y)\right| \mathrm{d} y+2 \int_{Q}\left|T\left(\left(b-b_{Q}\right) f_{1}\right)(y)\right| \mathrm{d} y \\
& +2|Q| \sup _{y \in Q}\left|T\left(\left(b-b_{Q}\right) f_{2}\right)(y)-T\left(\left(b-b_{Q}\right) f_{2}\right)\left(x_{Q}\right)\right| \\
= & I+I I+I I I .
\end{aligned}
$$

We estimate these terms respectively.

To the term $I$, for $x \in Q$, by Hölder's inequality and the part $(a)$ in Lemma 2.2, we have

$$
\begin{aligned}
I & \leq 2\left(\int_{Q}\left|b(y)-b_{Q}\right|^{q} \mathrm{~d} y\right)^{1 / q}\left(\int_{Q}|T f(y)|^{q^{\prime}} \mathrm{d} y\right)^{1 / q^{\prime}} \\
& \leq C|Q|^{\beta / n-1 / p+1 / q}|Q|^{1-1 / q}\|b\|_{\lambda_{\beta}^{p, q}}\left(|Q|^{-1} \int_{Q}|T f(y)|^{q^{\prime}} \mathrm{d} y\right)^{1 / q^{\prime}} \\
& \leq C|Q|^{1+\beta / n-1 / p}\|b\|_{\lambda_{\beta}^{p, q}} M_{q^{\prime}}(|T f|)(x) .
\end{aligned}
$$

To get $I I$, choosing $1<r<q$ such that $r q /(q-r)>1$, we firstly estimate $\left\|\left(b-b_{Q}\right) f_{1}\right\|_{r}$ as

$$
\begin{aligned}
\left\|\left(b-b_{Q}\right) f_{1}\right\|_{r}^{r}= & \int_{2 Q}\left|\left(b(y)-b_{Q}\right) f\right|^{r} \mathrm{~d} y \\
\leq & \left(\int_{2 Q}\left|b(y)-b_{Q}\right|^{q} \mathrm{~d} y\right)^{r / q}\left(\int_{2 Q}|f(y)|^{r q /(q-r)} \mathrm{d} y\right)^{(q-r) / q} \\
\leq & C|2 Q|^{r(\beta / n+1 / q-1 / p)}\|b\|_{\lambda_{\beta}^{p, q}}^{r} \\
& \times\left(|Q|^{-1} \int_{2 Q}|f(y)|^{r q /(q-r)} \mathrm{d} y\right)^{(q-r) / q}|Q|^{(q-r) / q} \\
\leq & C|Q|^{r(1 / r+\beta / n-1 / p)}\|b\|_{\lambda_{\beta}^{p, q}}^{r} M_{r q /(q-r)}^{r}(f)(x) .
\end{aligned}
$$

Take $r^{\prime}$ such that $1 / r+1 / r^{\prime}=1$. By the boundedness of Calderon-Zygmund Singular integral operator and the estimate of $\left\|\left(b-b_{Q}\right) f_{1}\right\|_{r}$ in $(2.4)$, we 
obtain

$$
\begin{aligned}
I I & =2 \int_{Q}\left|T\left(\left(b-b_{Q}\right) f_{1}\right)(y)\right| \mathrm{d} y \\
& \leq C\left\|T\left(\left(b-b_{Q}\right) f_{1}\right)\right\|_{r}|Q|^{1 / r^{\prime}} \\
& \leq C\left\|\left(\left(b-b_{Q}\right) f_{1}\right)\right\|_{r}|Q|^{1 / r^{\prime}} \\
& \leq C|Q|^{1 / r+\beta / n-1 / p}|Q|^{1 / r^{\prime}}\|b\|_{\lambda_{\beta}^{p, q}} M_{r q /(q-r)}(f)(x) \\
& \leq C|Q|^{1+\beta / n-1 / p}\|b\|_{\lambda_{\beta}^{p, q}} M_{r q /(q-r)}(f)(x) .
\end{aligned}
$$

Now we estimate III. Before we start the proof, we recall the smoothness condition of Calderon-Zygmund kernel, that is,

$$
|k(x-y)-k(-y)| \leq \frac{|x|}{|y|^{n+1}} .
$$

Then we have

$$
\begin{aligned}
& \left|T\left(\left(b-b_{Q}\right) f_{2}\right)(y)-T\left(\left(b-b_{Q}\right) f_{2}\right)\left(x_{Q}\right)\right| \\
& =\left|\int_{\mathbb{R}^{n}}\left(K(y-z)-K\left(x_{Q}-z\right)\right)\left(b(z)-b_{Q}\right) f_{2}(z) \mathrm{d} z\right| \\
& \leq C \int_{(2 Q)^{c}} \frac{\left|y-x_{Q}\right|}{\left|x_{Q}-z\right|^{n+1}}\left|b(z)-b_{Q}\right||f(z)| \mathrm{d} z \\
& \leq C \sum_{k=2}^{\infty} \int_{2^{k} Q \backslash 2^{k-1} Q} 2^{-k}\left|2^{k} Q\right|^{-1}\left|b(z)-b_{Q}\right||f(z)| \mathrm{d} z .
\end{aligned}
$$

It is easy to see that

$$
\begin{aligned}
\left|b_{Q}-b_{2 Q}\right| & \leq \frac{1}{|Q|} \int_{Q}\left|b(y)-b_{2 Q}\right| d y \\
& \leq \frac{2^{n}}{|2 Q|} \int_{2 Q}\left|b(y)-b_{2 Q}\right| d y \\
& \leq 2^{n}|2 Q|^{\beta / n-1 / p}\|b\|_{\lambda_{\beta}^{p, q}}
\end{aligned}
$$

and also

$$
\left|b_{2^{k} Q}-b_{Q}\right| \leq 2^{n} k\left|2^{k} Q\right|^{\beta / n-1 / p}\|b\|_{\lambda_{\beta}^{p, q}} .
$$


Thus by Hölder's inequality, (2.1) and (2.7), the last inequality in (2.6) is dominated by

$$
\begin{aligned}
& \sum_{k=2}^{\infty} 2^{-k}\left|2^{k} Q\right|^{-1} \int_{2^{k} Q \backslash 2^{k-1} Q}\left|b(z)-b_{Q}\right||f(z)| \mathrm{d} z \\
& \leq \sum_{k=2}^{\infty} 2^{-k}\left|2^{k} Q\right|^{-1}\left(\int_{2^{k} Q}\left|b(z)-b_{2^{k} Q}\right||f(z)| \mathrm{d} z+\int_{2^{k} Q}\left|b_{2^{k} Q}-b_{Q}\right||f(z)| \mathrm{d} z\right) \\
& \leq C \sum_{k=2}^{\infty} 2^{-k}\left|2^{k} Q\right|^{-1}\left(\int_{2^{k} Q}\left|b(z)-b_{Q}\right|^{q} \mathrm{~d} z\right)^{1 / q}\left(\int_{2^{k} Q}|f(z)|^{q^{\prime}} \mathrm{d} z\right)^{1 / q^{\prime}}+ \\
& \quad+C \sum_{k=2}^{\infty} 2^{-k} k\left|2^{k} Q\right|^{\beta / n-1 / p}\|b\|_{\lambda_{\beta}^{p, q}}\left|2^{k} Q\right|^{-1} \int_{2^{k} Q}|f(z)| \mathrm{d} z \\
& \leq C|Q|^{\beta / n-1 / p}\|b\|_{\lambda_{\beta}^{p, q}}\left(\sum_{k=2}^{\infty} 2^{k(-1+\beta-n / p)} M_{q^{\prime}}(f)(x)+\sum_{k=2}^{\infty} 2^{k(-1+\beta-n / p)} k M(f)(x)\right) \\
& \leq C|Q|^{\beta / n-1 / p}\|b\|_{\lambda_{\beta}^{p, q}}\left(M_{q^{\prime}}(f)(x)+M(f)(x)\right),
\end{aligned}
$$

where we use the assumption $0<\beta-n / p<1$. Thus we have

$$
I I I \leq C|Q|^{1+\beta / n-1 / p}\|b\|_{\lambda_{\beta}^{p, q}}\left(M_{q^{\prime}}(f)(x)+M(f)(x)\right) .
$$

Putting these estimates (2.3), (2.5) and (2.8) together, we obtain

$$
\begin{aligned}
& \frac{1}{|Q|^{1+\beta / n-1 / p}} \int_{Q}\left|T_{b} f(y)-\left(T_{b} f\right)_{Q}\right| \mathrm{d} y \\
& \quad \leq C\|b\|_{\lambda_{\beta}^{p, q}}\left(M_{q^{\prime}}(f)(x)+M_{q^{\prime}}(|T f|)(x)+M_{r q /(q-r)}(f)(x)+M(f)(x)\right) .
\end{aligned}
$$

Since $0<\beta-n / p<1, d>q /(q-1)=q^{\prime}$, we can choose $1<r$ and closely to 1 such that $d>r q /(q-r)>q /(q-1)$. Taking the supremum over all $Q$ such that $x \in Q$, and $L^{d}$ norm on both sides, and using Lemma 2.1, we conclude that

$$
\left\|T_{b} f\right\|_{\dot{F}_{d}^{(\beta-n / p), \infty}} \leq C\|b\|_{\lambda_{\beta}^{p, q}}\|f\|_{d}
$$

Thus, the proof of Theorem 1.1 is completed.

The proof of Theorem 1.2 is similar to that of Theorem 1.1.

Proof of Theorem 1.3 Fix $f \in L^{d}\left(\mathbb{R}^{n}\right)$ and recall that $x_{Q}$ denotes the center of a cube $\mathrm{Q}$, and $f_{1}=f \chi_{2 Q}, f_{2}=f-f_{1}$. Using Lemma 2.2(a) and 
the fact that $I_{b}^{\alpha} f-\left(I_{b}^{\alpha} f\right)_{Q}=I_{\left(b-b_{Q}\right)}^{\alpha} f-\left(I_{\left(b-b_{Q}\right)}^{\alpha} f\right)_{Q}$, we have

$$
\begin{aligned}
&\left\|I_{b}^{\alpha} f\right\|_{\dot{F}_{r}^{(\beta-n / p), \infty}} \approx C\left\|\sup _{Q \ni .} \frac{1}{|Q|^{(1+\beta / n-1 / p)}} \int_{Q}\left|I_{b-b_{Q}}^{\alpha} f-\left(I_{b-b_{Q}}^{\alpha} f\right)_{Q}\right|\right\|_{r} \\
& \leq C \| \sup _{Q \ni .} \frac{1}{|Q|^{(1+\beta / n-1 / p)}} \int_{Q} \mid I_{b-b_{Q}}^{\alpha} f-\left(I^{\alpha}\left(\left(b-b_{Q}\right) f_{2}\right)\left(x_{Q}\right) \mid \|_{r}\right. \\
& \leq C\left\|\sup _{Q \ni .} \frac{1}{|Q|^{(1+\beta / n-1 / p)}} \int_{Q}\left|\left(b-b_{Q}\right) I^{\alpha} f\right|\right\|_{r}+ \\
&+C\left\|\sup _{Q \ni .} \frac{1}{|Q|^{(1+\beta / n-1 / p)}} \int_{Q}\left|I^{\alpha}\left(\left(b-b_{Q}\right) f\right)-I^{\alpha}\left(\left(b-b_{Q}\right) f_{2}\left(x_{Q}\right)\right)\right|\right\|_{r} \\
& \leq C\left\|\sup _{Q \ni .} \frac{1}{|Q|^{(1+\beta / n-1 / p)}} \int_{Q}\left|\left(b-b_{Q}\right) I^{\alpha} f\right|\right\|_{r}+ \\
&+\left\|\sup _{Q \ni .} \frac{1}{|Q|^{1+(\beta+\alpha) / n-1 / p}} \int_{Q}\left|I^{\alpha}\left(\left(b-b_{Q}\right) f\right)-I^{\alpha}\left(\left(b-b_{Q}\right) f_{2}\left(x_{Q}\right)\right)\right|\right\|_{d} .
\end{aligned}
$$

Since $1<d<r<\infty, 0<\beta-n / p<1,1 / d-1 / r=\alpha / n$. The last inequality follows from the following lemma, with $\beta-n / p$ in the place of $\gamma$.

Lemma 2.3. ([8] pp.71-72) Let $1<d<r<\infty, 1 / d-1 / r=\alpha / n$. Suppose that for each cube $Q$ we have a function $h^{Q}$, defined on this cube. Then, for $\gamma \geq 0$,

$$
\left\|\sup _{Q \ni .} \frac{1}{|Q|^{1+\gamma / n}} \int_{Q}\left|h^{Q}\right|\right\|_{r} \leq C\left\|\sup _{Q \ni .} \frac{1}{|Q|^{1+\gamma / n+\alpha / n}} \int_{Q}\left|h^{Q}\right|\right\|_{d},
$$

where the constant $C$ depends only on $d, r, \alpha$ and $n$.

Lemma 2.3, as in [8], specifies that the function $h^{Q}$ have the form $h-h^{Q}$, but the proof carries over immediately to the more general case above. Thus,

$$
\begin{aligned}
\left\|I_{b}^{\alpha} f\right\|_{\dot{F}_{r}^{(\beta-n / p), \infty}} \leq & C\left\|\sup _{Q \ni .} \frac{1}{|Q|^{(1+\beta / n-1 / p)}} \int_{Q}\left|\left(b-b_{Q}\right) I^{\alpha} f\right|\right\|_{r} \\
& +C\left\|\sup _{Q \ni .} \frac{1}{|Q|^{(1+(\alpha+\beta) / n-1 / p)}} \int_{Q}\left|I^{\alpha}\left(\left(b-b_{Q}\right) f_{1}\right)\right|\right\|_{d} \\
& +C \| \sup _{Q \ni .} \frac{1}{|Q|^{((\alpha+\beta) / n-1 / p)}} \times \\
& \times \sup _{y \in Q}\left|I^{\alpha}\left(\left(b-b_{Q}\right) f_{2}\right)(y)-I^{\alpha}\left(\left(b-b_{Q}\right) f_{2}\right)\left(x_{Q}\right)\right| \|_{d} \\
\leq & I+I I+I I I .
\end{aligned}
$$


Using Lemma $2.2(a)$, we can estimate $I$ as follows

$$
\begin{aligned}
\int_{Q}\left|b(y)-b_{Q}\right|\left|I^{\alpha} f(y)\right| \mathrm{d} y & \leq C\left(\int_{Q}\left|b(y)-b_{Q}\right|^{q} \mathrm{~d} y\right)^{1 / q}\left(\int_{Q}\left|I^{\alpha} f(y)\right|^{q^{\prime}} \mathrm{d} y\right)^{1 / q^{\prime}} \\
& \leq C|Q|^{1+\beta / n-1 / p}\|b\|_{\lambda_{\beta}^{p, q} M_{q^{\prime}}\left(I^{\alpha} f\right)(x) .}
\end{aligned}
$$

Since $r>q^{\prime}$, we have

$$
\begin{aligned}
I & \leq C\|b\|_{\lambda_{\beta}^{p, q}}\left\|M_{q^{\prime}}\left(I^{\alpha} f\right)\right\|_{r} \\
& \leq C\|b\|_{\lambda_{\beta}^{p, q}}^{p,}\left\|I^{\alpha} f\right\|_{r} \\
& \leq C\|b\|_{\lambda_{\beta}^{p, q}}\|f\|_{d} .
\end{aligned}
$$

We now estimate $I I$. Choose $1<h<q$, and $\bar{h}$ such that then $(1 / h)-(1 / \bar{h})=\alpha / n$. Consider $\left\|\left(b-b_{Q}\right) f_{1}\right\|_{h}$ as

$$
\begin{aligned}
& \left(\int_{Q}\left|b(y)-b_{Q}\right|^{h}\left|f_{1}(y)\right|^{h} \mathrm{~d} y\right)^{1 / h} \\
& \quad \leq\left(\int_{Q}\left|b(y)-b_{Q}\right|^{q} \mathrm{~d} y\right)^{1 / q}\left(\int_{Q}\left|f_{1}(y)\right|^{h q /(q-h)} \mathrm{d} y\right)^{(1 / h-1 / q)} \\
& \quad \leq C|Q|^{(\beta / n+1 / q-1 / p)}\|b\|_{\lambda_{\beta}^{p, q}} M_{q h /(q-h)}(f)(x)|Q|^{1 / h-1 / q} \\
& \quad \leq C|Q|^{(\beta / n+1 / h-1 / p)}\|b\|_{\lambda_{\beta}^{p, q}} M_{q h /(q-h)}(f)(x) .
\end{aligned}
$$

Thus,

$$
\begin{aligned}
& \frac{1}{|Q|^{(1+(\alpha+\beta) / n-1 / p)}} \int_{Q}\left|I^{\alpha}\left(\left(b-b_{Q}\right) f_{1}\right)(y)\right| \mathrm{d} y \mid \\
& \leq \frac{1}{|Q|^{(1+(\alpha+\beta) / n-1 / p)}}\left\|I^{\alpha}\left(\left(b-b_{Q}\right) f_{1}\right)\right\|_{\bar{h}}|Q|^{1-1 / \bar{h}} \\
& \leq C|Q|^{-1-\alpha / n-\beta / n+1-1 / \bar{h}+1 / p}\left\|\left(b-b_{Q}\right) f_{1}\right\|_{h} \\
& =C|Q|^{-1 / h-\beta / n+1 / p}\left\|\left(b-b_{Q}\right) f_{1}\right\|_{h} . \\
& \leq C\|b\|_{\lambda_{\beta}^{p, q}} M_{q h /(q-h)}(f)(x),
\end{aligned}
$$

Since $d>q /(q-1)$, we can choose $1<h$ such that $d>q h /(q-h)>$ $q /(q-1)$, and obtain

$$
\begin{aligned}
I I & \leq C\|b\|_{\lambda_{\beta}^{p, q}}\left\|M_{q h /(q-h)}(f)\right\|_{d} \\
& \leq C\|b\|_{\lambda_{\beta}^{p, q}\|f\|_{d} .}
\end{aligned}
$$


Now we estimate III. Set $y \in Q$. By (2.7) and Lemma 2.2, we have

$$
\begin{aligned}
& \frac{1}{|Q|^{(\alpha+\beta) / n-1 / p}}\left|I^{\alpha}\left(\left(b-b_{Q}\right) f_{2}\right)(y)-I^{\alpha}\left(\left(b-b_{Q}\right) f_{2}\right)\left(x_{Q}\right)\right| \\
\leq & \frac{C}{|Q|^{(\alpha+\beta) / n-1 / p}} \int_{(2 Q)^{c}} \frac{\left|y-x_{Q}\right|}{\left|x_{Q}-z\right|^{n+1-\alpha}}\left|b(z)-b_{Q}\right||f(z)| \mathrm{d} z \\
\leq & \frac{C}{|Q|^{(\alpha+\beta) / n-1 / p}} \sum_{k=2}^{\infty} \int_{2^{k} Q \backslash 2^{k-1} Q} 2^{-k}\left|2^{k} Q\right|^{-1+\alpha / n}\left|b(z)-b_{2^{k} Q}\right||f(z)| \mathrm{d} z \\
\leq & \frac{C}{|Q|^{(\alpha+\beta) / n-1 / p}}\left(\sum_{k=2}^{\infty} 2^{-k}\left|2^{k} Q\right|^{-1+\alpha / n} \int_{2^{k} Q}\left|b(z)-b_{2^{k} Q}\right||f(z)| \mathrm{d} z+\right. \\
& \left.+\sum_{k=2}^{\infty} 2^{-k} k\left|2^{k} Q\right|^{(\alpha+\beta) / n-1 / p}\|b\| \dot{\wedge}_{\beta}^{p, q}\left|2^{k} Q\right|^{-1} \int_{2^{k} Q}|f(z)| \mathrm{d} z\right) \\
\leq & \frac{C\|b\| \dot{\lambda}_{\beta}^{p, q}}{|Q|^{(\alpha+\beta) / n-1 / p}}\left(\sum_{k=2}^{\infty} 2^{-k}\left|2^{k} Q\right|^{(\alpha+\beta) / n-1 / p} M_{q^{\prime}}(f)(x)+\right. \\
& \left.+\sum_{k=2}^{\infty} 2^{-k} k\left|2^{k} Q\right|^{(\alpha+\beta) / n-1 / p} M(f)(x)\right) \\
\leq & C\|b\| \dot{\wedge}_{\beta}^{p, q}\left(\sum_{k=2}^{\infty} 2^{k(-1+\alpha+\beta-n / p)} M_{q^{\prime}}(f)(x)+\right. \\
& \left.+\sum_{k=2}^{\infty} 2^{k(-1+\alpha+\beta-n / p)} k M(f)(x)\right) \\
\leq &
\end{aligned}
$$

where we first assume that $\alpha+\beta-n / p<1$. Thus,

$$
\begin{aligned}
I I I & \leq C\|b\|_{\dot{\lambda}_{\beta}^{p, q}}\left(\left\|M_{q^{\prime}}(f)\right\|_{d}+\|M(f)\|_{d}\right) \\
& \leq C\|b\|_{\dot{\lambda}_{\beta}^{p, q}}\|f\|_{d} .
\end{aligned}
$$

Putting (2.9), (2.10) and (2.11) together, Theorem 1.3 is proved under the assumption $\alpha+\beta-n / p<1$. Following the facts 1.9 and 1.10 in [6] on pages 10 and 11, we can also get the result when $0<\alpha<n$.

The proof of the Theorem 1.4 is similar to that of Theorem 1.3, and we omit the details. 


\section{References}

[1] S. Chanillo, A note on commutators, Indiana Univ. Math. J., 31 (1982), $7-16$.

[2] Y. Chen and B. Ma, The boundedness of higher order commutators with Besov functions, Beijing Shifan Daxue Xuebao(Chinese), 42 (2006), 3234.

[3] R. Coifman, R. Rochberg and G. Weiss, Facorization theorems for Hardy spaces in several variables, Ann. of Math., 103 (1976), 611-635.

[4] R. Devore and R. Sharpley, Maximal functions measuring smoothness, Mem. Amer. Math. Soc., (47) 1984.

[5] S. Janson, Mean oscillation and commutators of singular integral operators, Ark. Math., 16 (1978), 263-270.

[6] M. Paluszyński, Characterization of the Besov spaces via the commutator operator of Coifman, Rochberg and Weiss, Indiana Univ. Math. J., 44 (1995), 1-17.

[7] C. Pérez, R. Trujillo-González, Sharp weighted estimates for multilinear commutators, J. London Math. Soc., 65 (2002), 672-692.

[8] A. Seeger, A note on Triebel-Lizorkin spaces, Approximation and Functions, PWN, Warsaw, 1989.

[9] E. M. Stein, Singular Integrals and Defferentialbility Properties of Functions, Princeton Univ. Press, Priceton, NJ, 1970.

[10] H. Triebel, Theory of Function Spacs, Besel Boston, Birkhauser,1983.

College of Mathematics and Information Engineering

Jiaxing University

Zhejiang, 314001

P. R. China

(E-mail : gaoxionglue@126.com)

(E-mail : blma@mail.zjxu.edu.cn)

(Received : February 2009) 


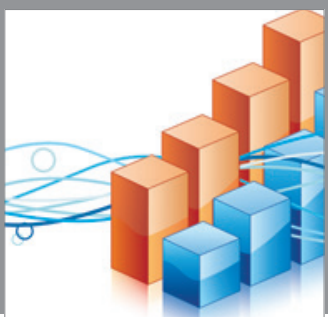

Advances in

Operations Research

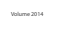

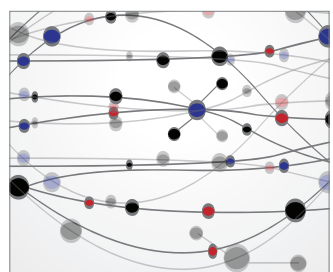

\section{The Scientific} World Journal
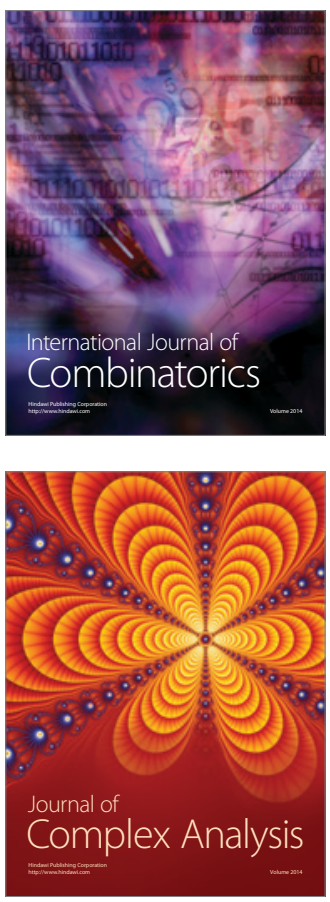

International Journal of

Mathematics and

Mathematical

Sciences
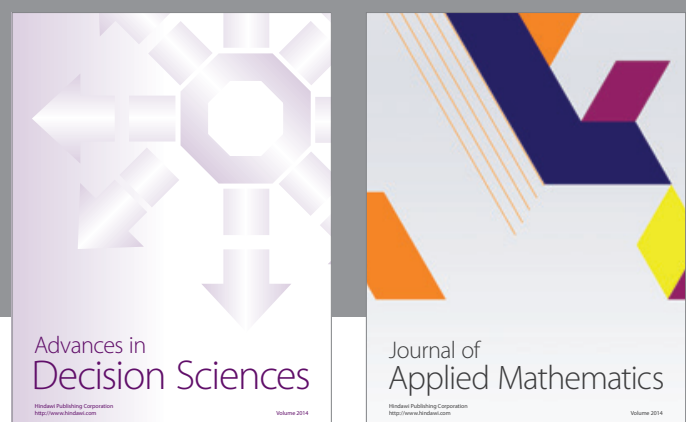

Journal of

Applied Mathematics
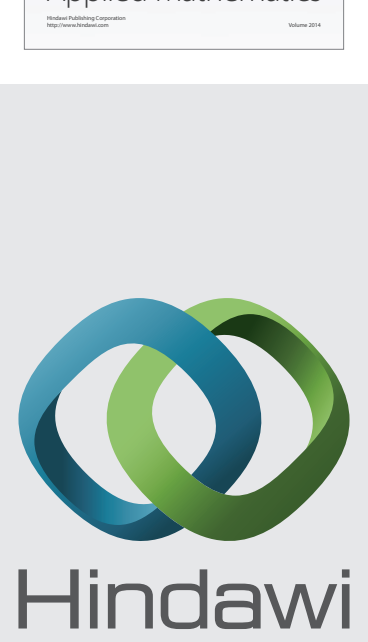

Submit your manuscripts at http://www.hindawi.com
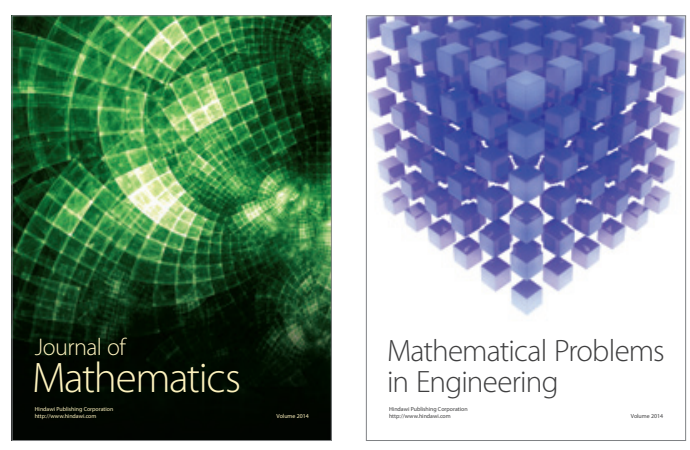

Mathematical Problems in Engineering
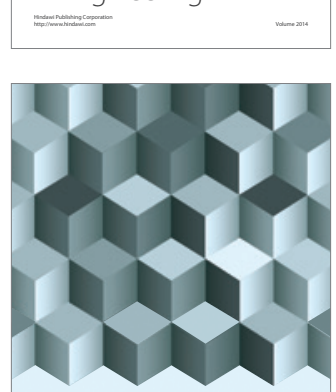

Journal of

Function Spaces
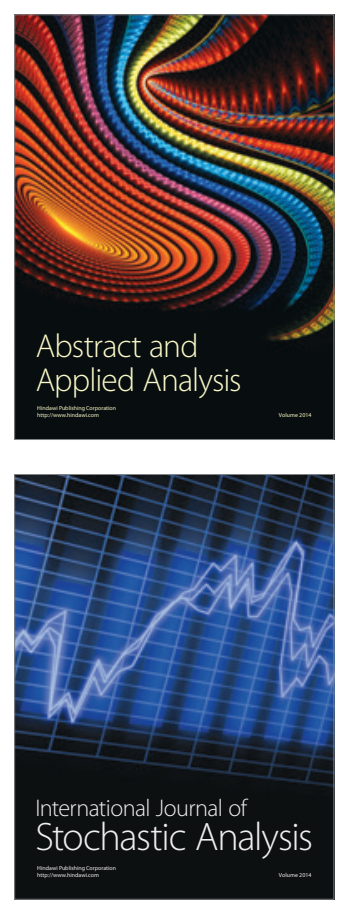

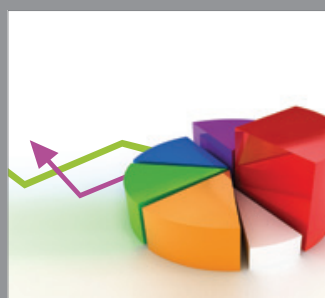

ournal of

Probability and Statistics

Promensencen
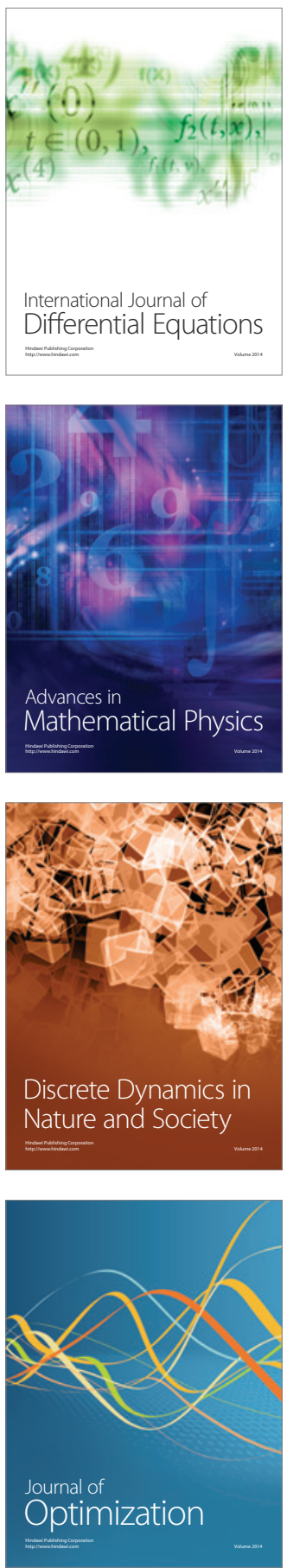